\title{
Production of Ultrashort FEL XUV Pulses via a Reverse Undulator Taper *
}

\author{
W. M. Fawley ${ }^{1}$ \\ Center for Beam Physics, Lawrence Berkeley National Laboratory, Berkeley, CA 94720, USA
}

\begin{abstract}
We adapt the "reverse taper" scheme presented by Saldin et al. (Phys. Rev. ST Accel. Beams, 9, 050702 [2006]) for attosecond pulse production to the XUV/soft-xray regime. We find that that GW-level pulses of a few femtosecond duration or shorter can be produced using electron beams of quite moderate parameters and undulators of 20 -m length or shorter. The output pulse is significantly shifted in wavelength relative to the main background which permits a further increase in contrast ratio via simple monochromatization. Moreover, the output pulse has a natural wavelength chirp that allows further temporal compression, if wanted. Both positive and negative chirps can be produced depending upon the sign of the undulator taper.
\end{abstract}

Key words: free-electron laser, ultrashort pulse production PACS: 41.50.+h, 41.60.Cr, 42.55.Vc, 52.59.-f

\section{Introduction}

The past two decades have seen impressive achievements in the production of coherent, ultrashort (i.e., femtosecond and shorter durations) radiation pulses from optical to x-ray wavelengths. These pulses have facilitated the birth and rapid development of "ultrafast science" that includes studies of non-equilibrium processes, etc. Concurrently, there has been similar development in expanding the operating wavelength range of free-electron lasers, with the FLASH facility [1] currently reaching down to $12 \mathrm{~nm}$ and the LCLS facility [2] projected to obtain hard x-ray pulses at wavelengths as short as $0.15 \mathrm{~nm}$ by the end of 2009. In recent years, FEL researchers have explored a number of ways to produce high-power, ultrashort pulses at XUV and shorter wavelengths with a particular emphasis

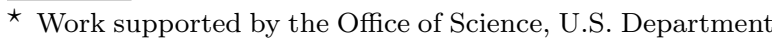
of Energy under Contract No. DE-AC02-05CH11231.

1 WMFawley@lbl.gov
}

on temporal synchronism with external lasers to facilitate pump-probe experiments.

Many short pulse schemes rely upon manipulating one or more properties of an ultrashort temporal portion of a much longer e-beam, such as transverse emittance [3], energy [4][5][6][7] or current [8]. These manipulations generally use a few-cycle, nearIR laser pulse to energy-modulate the e-beam so that, when combined with other transport elements such as chromatic chicanes, foils, specially-tuned radiator undulators, the FEL emission will predominately arise from the modulated portion.

Saldin et al. [7] cleverly combined the above rapid energy modulation together with a positive undulator strength taper in $z$ to produce high peak power, sub-fs pulses in the hard x-ray regime. As will be shown in the remainder of this paper, the scheme scales well to much longer wavelengths (i.e., soft X-ray and XUV). In $\S I I$ we review the underlying physics of the scheme and some of the expected properties of the output pulse. We then apply the scheme 
to the XUV regime and present simulation results in $\S I I I$ for both SASE and seeded cases. In $\S$ IV we then examine more closely the characteristic wavelength chirp of the output pulse and its possible control.

\section{Review of the Reverse-Taper Approach to Short Pulse Production}

The idea that changing the resonant electron energy $\gamma_{R} m c^{2}$ with $z$ would affect total SASE emission has been known for a number of years, as has the knowledge that undulator tapering could counteract external energy losses from wakefields. Nuhn [9] noticed that LCLS SASE simulations with longitudinal wakefield losses showed greater radiation emission on the temporal portions where $d \gamma / d z$ was positive relative to the equivalent negative regions. Reiche (private communication) showed the combination of a time-dependent wakefield loss together with an undulator taper would localize intense SASE emission to a temporal region where the net change in $\gamma_{R}$ was close to zero. Later, Huang and Stupakov [10] analytically showed that the optimal net energy loss rate was actually not zero but slightly positive:

$$
\left.\frac{d \gamma}{d z}\right|_{o p t} \approx 0.2 \gamma_{0} \frac{4 \pi \rho}{\lambda_{w}}
$$

where $\lambda_{w}$ is the undulator wavelength and $\rho$ is the FEL (or Pierce) parameter [11]. At this optimum, the nonlinear energy extraction efficiency is about twice that of the untapered case.

Concurrently with these findings, researchers developed a number of schemes relying upon high power, few-cycle intense lasers to produce extremely short duration FEL output pulses at soft [4] and hard [5],[6] x-ray wavelengths. In general, these schemes depend upon energy modulation of the electron beam by the external laser in a short undulator and subsequent resonant tuning of a downstream radiator to the peak energy of the modulation, leading to an output pulse duration much less than the modulation period $\lambda_{M} / c$.

More recently, Saldin et al. [7] combined many of the above ideas and observations into a new scheme that uses "normal" FEL slippage together with a strong, temporally localized $d \gamma / d t$ produced by a few cycle optical laser to produce an equivalent $d \gamma / d z$ (as seen by the radiation) that could then be compensated by a taper with $z$ of the RMS undulator strength parameter $a_{w}$. When $d \gamma / d t$ is extremely large, one need only satisfy $(d \gamma / c d t) \times \beta_{\text {slip }} \approx$ $\left(d \gamma_{R} / d a_{w}\right) \times d a_{w} / d z$ or, equivalently,

$$
\frac{d \ln a_{w}}{d z}=-\frac{\lambda_{s}}{\lambda_{w}} \frac{\left(1+a_{w}^{2}\right)}{a_{w}^{2}} \frac{1}{c} \frac{d \ln \gamma}{d t}
$$

Those portions of the electron beam with much smaller $d \gamma / d t$ will have a much reduced or nonexistent gain. For modulation phases producing a strongly negative $d \gamma / d t$ such that the FEL radiation slips forward toward higher electron beam energy, the compensating positive $d a_{w} / d z$ can produce a negative gain where $d \gamma / d t \approx 0$, thus leading to enhanced contrast between the strongly modulated portion and the remainder of the electron beam. However, even in the alternative case of a strongly positive $d \gamma / d t$ and a negative undulator taper, one still expects good contrast because of the narrow effective width of the gain curve.

As noted in Ref. [7], the energy chirp leads to a corresponding chirp in the output radiation pulse. The fact that one can change the magnitude and sign of the energy chirp by manipulating the strength and phase of the few-cycle optical laser pulse indicates a tool by which to vary the strength and sign of the output chirp, a subject we explore more in $\S \mathrm{IV}$.

\section{Application to XUV Wavelengths}

In this section we first discuss the electron beam, modulating laser, undulator, and relevant numerical simulation parameters that were adopted for this study of ultrashort XUV pulse production. We then present simulation results for both SASE- and external seeding-initiated cases at 8-nm wavelength.

\subsection{Physical and Simulation Parameters}

For this particular study we adopted electron beam parameters corresponding to an ongoing study at LBNL for a future light source that would operate in the XUV to soft x-ray wavelength range. As shown in Table 1, the adopted electron beam has moderate energy, very high brightness (in terms of transverse emittance) and moderate $\left(\sim 10^{-4}\right.$ normalized) incoherent energy spread. While results (in terms of final power) at wavelengths $\geq 8 \mathrm{~nm}$ are reasonably insensitive to such a low emittance, short wavelength (e.g., $\left.\lambda_{s}=2 \mathrm{~nm}\right)$ results are sensitive. A relatively long wavelength $\left(\lambda_{M}=2.2 \mu \mathrm{m}\right)$ for the energy modulation gives a better match to the XUV region when compared with the $800-\mathrm{nm}$ 
Table 1

Electron Beam and Modulating Laser Parameters

\begin{tabular}{|c|c|c||c|c|c|}
\hline current & 1.0 & $\mathrm{kA}$ & energy & 2.0 & $\mathrm{GeV}$ \\
\hline$\varepsilon_{n}$ & 0.5 & $\mathrm{~mm}-\mathrm{mrad}$ & $\sigma_{E}$ & 200 & $\mathrm{keV}$ \\
\hline \hline$\lambda_{M}$ & 2200 & $\mathrm{~nm}$ & $E_{M}$ & \pm 7.5 & $\mathrm{MeV}$ \\
\hline$\sigma_{t}$ (damping) & 5.85 & $\mathrm{fs}$ & mod. phase & $\pi$ & radians \\
\hline \hline
\end{tabular}

wavelength chosen by Saldin et al. for their hard xray studies. Essentially, one accepts a a somewhat greater output radiation pulse duration in order to obtain more rapid growth in $z$ and a larger output pulse energy. The $\pm 7.5 \mathrm{MeV}$ modulation is $4 \mathrm{X}$ smaller than that adopted in Ref. [4] and is obtainable with commercial lasers today. We presume that the output phase of the few-cycle laser can be well controlled; shot-to-shot phase jitter of more than a couple tenths of a radian would likely be problematical because the peak value of $d \gamma / d t$ would no longer be matched to the undulator taper.

To study the utility of the taper/chirped-energy scheme in the XUV, we did a series of numerical simulations with the GINGER code [12] for both SASE and external seeding configurations. Twenty-five or greater runs with different shot noise random number seeds were performed for each SASE case to obtain reasonable statistics. Typically, temporal resolution of 80 attoseconds or less was used together with a total time window of at least 5 optical modulation periods. Each slice had 4096 macroparticles. Total slippage over the full undulator length was normally less than half the simulation window; this prevented anomalous numerical effects arising from the imposition of periodic boundary conditions in time. We adopted perfect energy modulation from the few-cycle external laser following the relation

$\Delta E(t)=E_{M} \sin \left(\phi_{M}+\frac{2 \pi c t}{\lambda_{M}}\right) \times \exp \left[\frac{-\left(t-t_{o}\right)^{2}}{2 \sigma_{t}^{2}}\right]$

The undulator had linear polarization, fixed period, and a simple linear taper for $a_{w}(z)$ beginning at its entrance $(z \equiv 0)$. In addition to "normal" undulator curved pole tip focusing, an additional (harmonic) focusing term equivalent to $k_{\beta}=(2 \pi / 25) \mathrm{m}^{-1}$ was presumed in each transverse plane, leading to a matched RMS beam size of $\approx 30 \mu \mathrm{m}$. Experimentally, similar focusing could be provided with a quadrupole FODO lattice. According to the empirical M. Xie formula [13], the FEL parameter $\rho \approx 2.9 \times 10^{-3}$ for $\lambda_{s}=8 \mathrm{~nm}$ and $\lambda_{w}=3 \mathrm{~cm}$.

In order to use the same $E_{M}$ and $a_{w}(z)$, the ra- tio $\left(\lambda_{s} / \lambda_{w}\right)$ was kept constant (see Eq. 2) with $\lambda_{w}$ growing from $15 \mathrm{~mm}$ at $4 \mathrm{~nm}$ to $120 \mathrm{~mm}$ at $32 \mathrm{~nm}$. For $E_{M}=7.5 \mathrm{MeV}$ and $a_{w}=2.7$, we empirically found that the "best" (particularly in terms of contrast) undulator taper was 3.5\%/10 m; Eq. 3 would predict $3.25 \%$. For $\lambda_{s}=2 \mathrm{~nm}, \lambda_{w}$ remained $15 \mathrm{~mm}$ and the normalized taper was reduced to $2 \%$ per $10 \mathrm{~m}$ with the initial $a_{w}=1.75$ For the SASE cases at $\lambda_{s}=32 \mathrm{~nm}$, to improve gain we reduced $\lambda_{w}$ to $6 \mathrm{~cm}$, the taper was increased to $5 \%$ per $10 \mathrm{~m}$ and $E_{M}$ reduced to $4.5 \mathrm{MeV}$.

\subsection{SASE Cases}

Adopting the beam, external laser, and undulator parameters mentioned $\mathrm{n}$ the previous section, we did a series of SASE-initiated runs at various wavelengths. As a representative (single shot) case, Fig. 1 shows the instantaneous near-field radiation power, the macroparticle microbunching, and the spectrum of the on-axis far field. One sees that the contrast ratio for the power is extremely good and that nearly all the output power is contained within a 3-fs region. By contrast, the bunching has a temporally wider central "spike" and there are similar spikes at half the amplitude 7-fs away (i.e., one external laser period). The spectral power has a FWHM of about $0.12 \mathrm{~nm}$ and is offset $\sim 0.5 \mathrm{~m}$ from the initial $(z=$ $0 \mathrm{~m}$ ) resonance of $8.0 \mathrm{~nm}$.

For more insight into the time-frequency "phase space" of the radiation, Wigner transforms $W(\omega, t)$ of the on-axis, far field were calculated where

$$
W(\omega, t) \equiv \int d \tau E\left(t-\frac{\tau}{2}\right) E^{*}\left(t+\frac{\tau}{2}\right) e^{+i \omega \tau}
$$

Figure 2 displays false-color images of $W$ at three different $z$ locations. It is obvious both that the pulse is highly localized both in time and wavelength, that with increasing $z$ the centroid in wavelength moves redwards, and that there is a distinct chirp with positive $d \lambda / d t$.

A series of 64 separate GINGER runs distinguished by different random number seeds for shot noise initialization were done to extract average pulse waveforms and the shot-to-shot deviations (see Fig. 3). Plots of $P(t)$ and pulse energy $\varepsilon_{R}$ as a function of $z$ show the large deviations one expects for SASE when it is composed of only one or two longitudinal modes; nonetheless the FWHM for the output pulse duration remains less than 2 fs with a peak power of just under $800 \mathrm{MW}$. The large deviations of $\varepsilon_{R}$ indicate fluctuations in the overall 

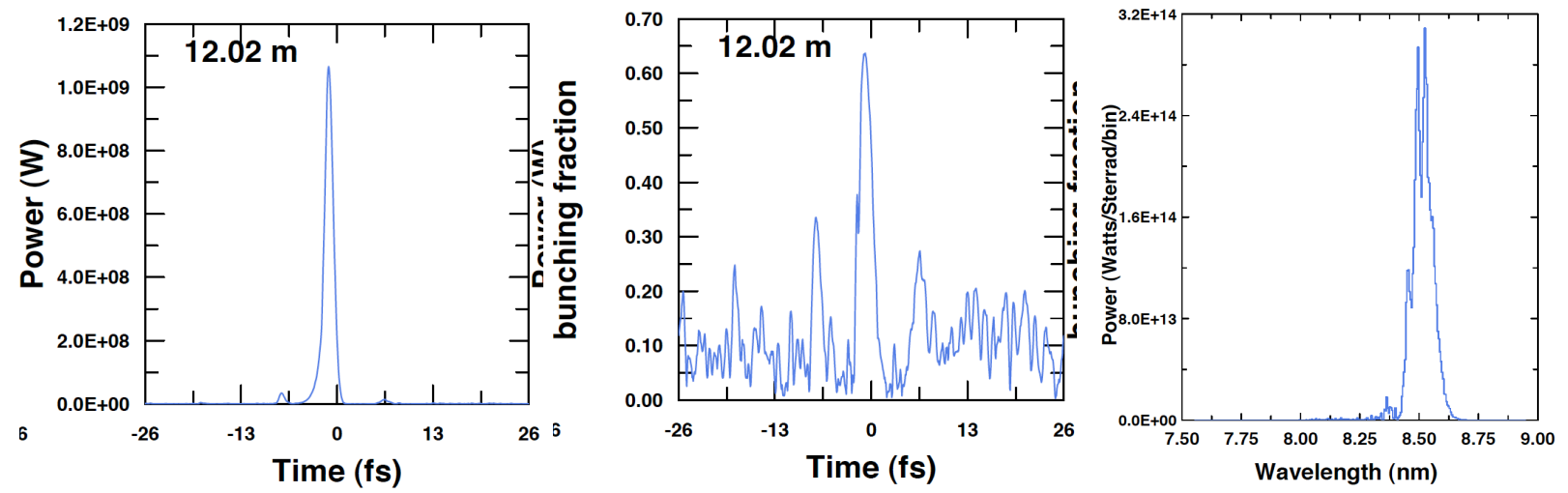

Fig. 1. Instantaneous power, bunching, and on-axis far field spectrum at undulator exit $(z=12 \mathrm{~m})$ for a particular SASE run initially resonant at $8.0-\mathrm{nm}$ wavelength.
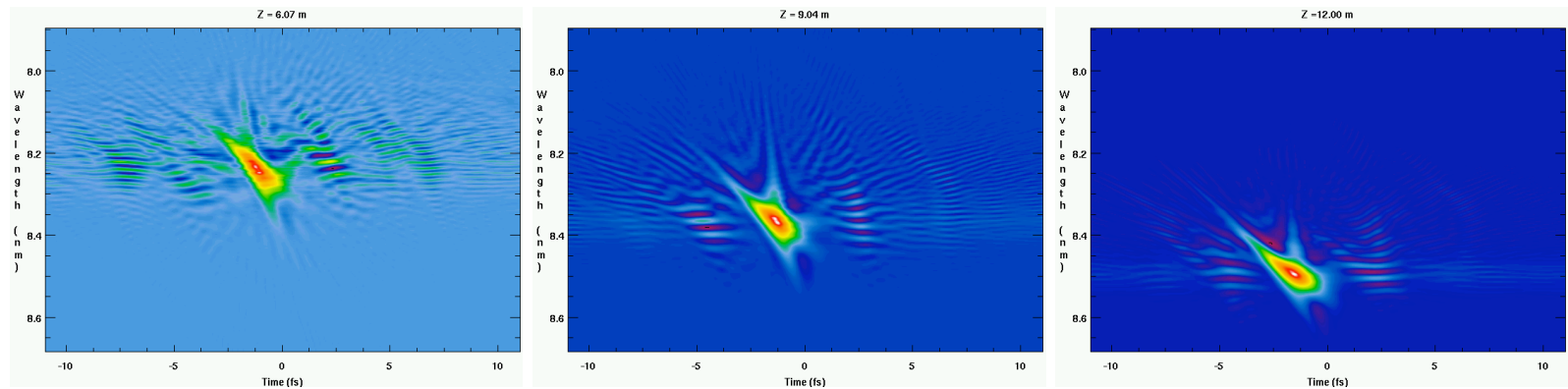

Fig. 2. False color images of the Wigner transform of the far field, on-axis radiation emission at distances of 6,9 , and $12 \mathrm{~m}$ into the undulator for the 8-nm SASE case of Fig. 1.
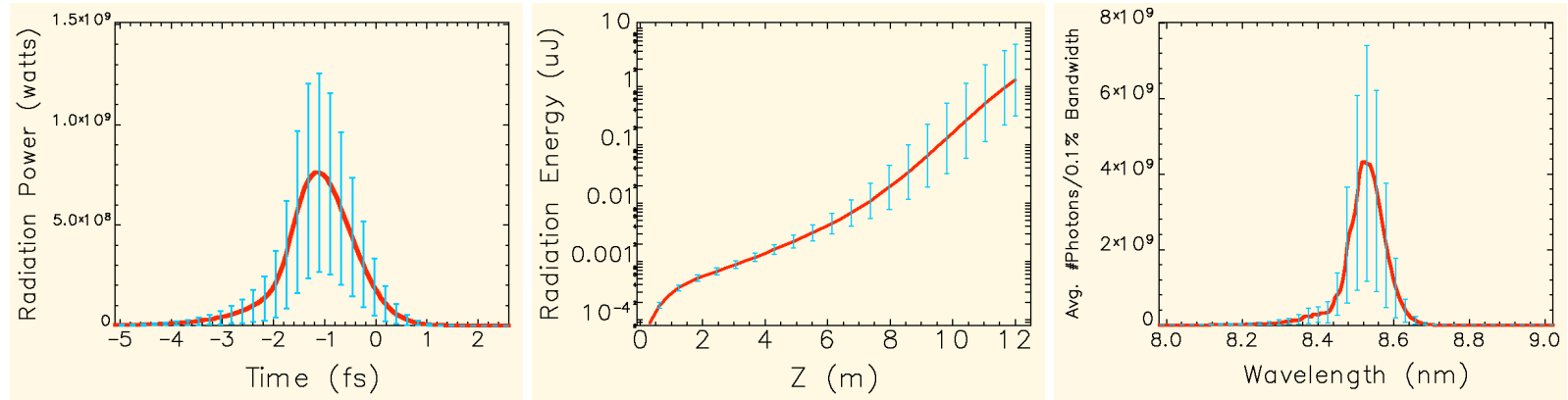

Fig. 3. Average and RMS standard deviations of the instantaneous power, integrated radiation pulse energy $\varepsilon_{R}$, and spectra from analysis of 64 independent SASE 8-nm runs.

integrated power underly those in $P(t)$ and $P(\omega)$, rather than an alternate explanation of a single spike (with similar integrated $\varepsilon_{R}$ ) jumping around shot-to-shot $\sim \pm 0.5 \mathrm{fs}$ in time and $\sim \pm 0.5 \mathrm{~nm}$ in central wavelength. Thus, experimentally, while it will be necessary to monitor the overall radiation energy on a shot-to-shot basis, fs-level temporal synchronization in pump-probe experiments should be possible even for SASE-initiated configurations.

\subsection{Seeded Cases}

Simulations for the reverse taper scheme seeded by an external laser were done over a wavelength range from 2 to $32 \mathrm{~nm}$. Here we present some results with a 8.0-nm external seed at a power of $5 \mathrm{MW}$, and a waist size of 35 microns (Rayleigh range $=0.47 \mathrm{~m}$ ) at undulator entrance. As shown by the top row of plots in Fig. 4, the near-field power increases to a peak above $600 \mathrm{MW}$ by $z=8 \mathrm{~m}$ centered in a spike 

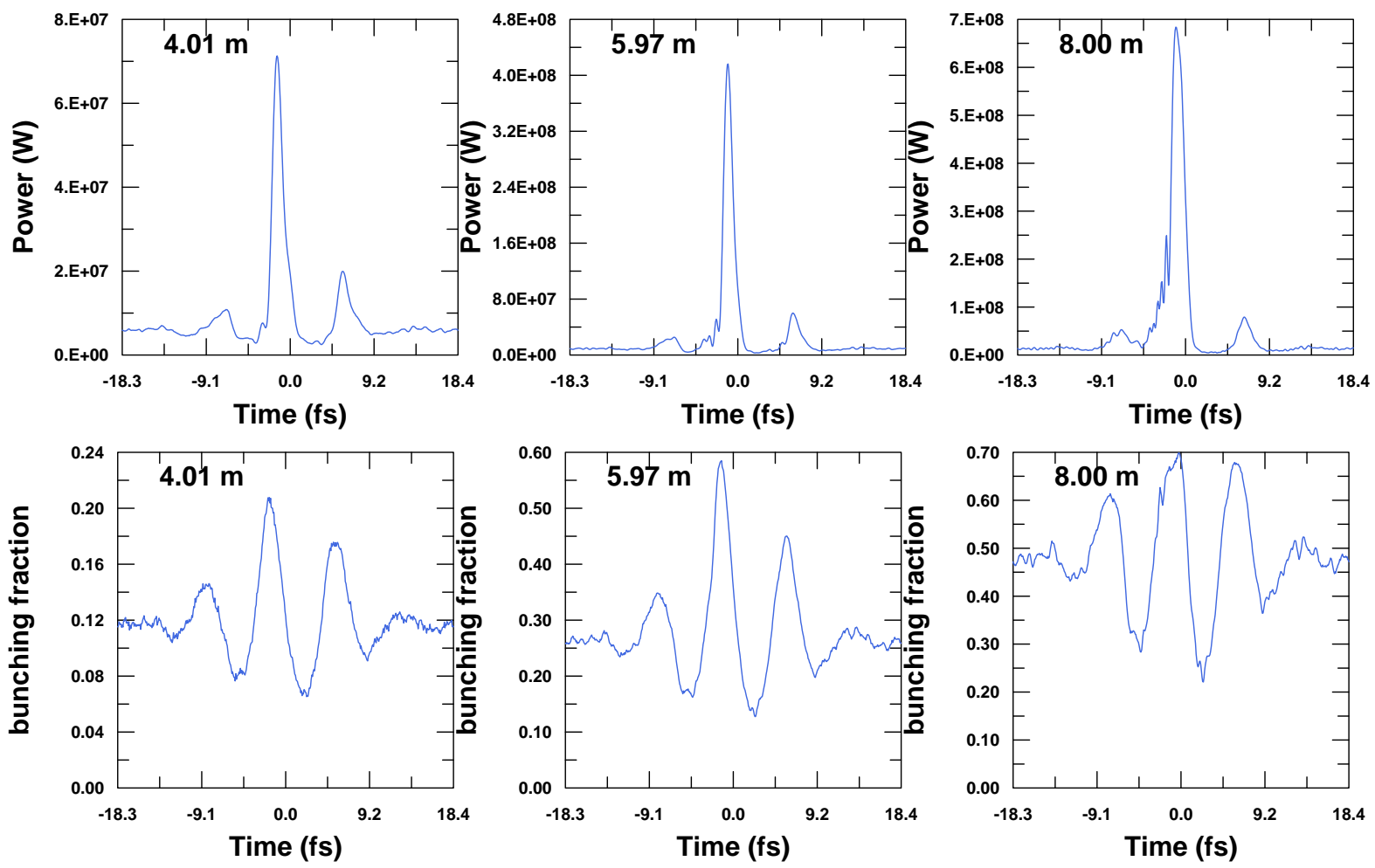

Fig. 4. Snapshots of instantaneous power and microbunching fraction for a seeded 8-nm case at 3 different z-locations.
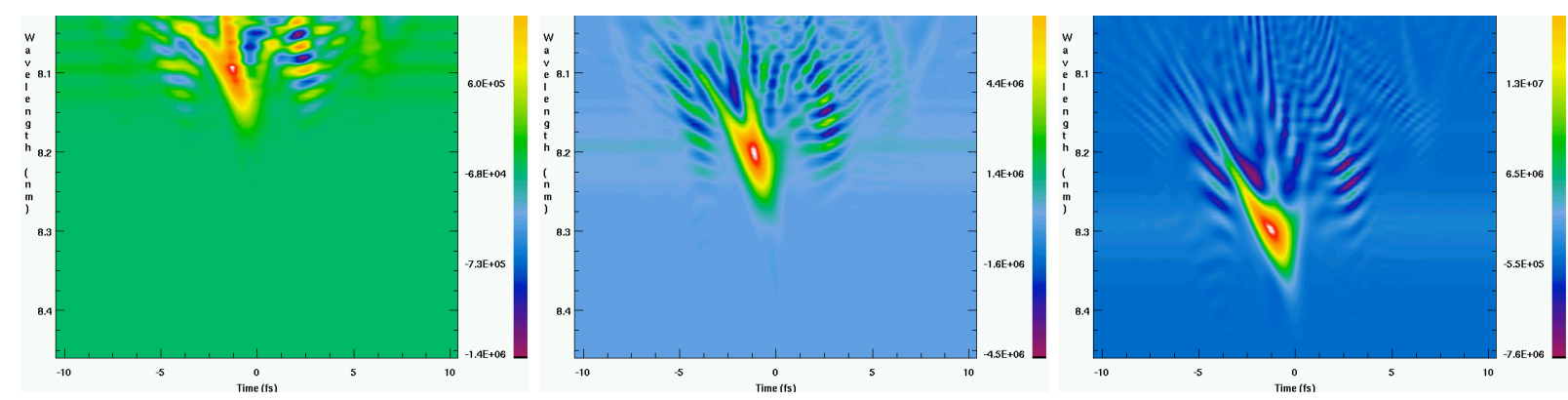

Fig. 5. Wigner transform of the far field, on-axis radiation emission at distances of 4,6 , and $8 \mathrm{~m}$ in the undulator for a case seeded by a 5-MW external laser at 8.2 -nm wavelength.

with a FWHM less than 1.5 fs. The contrast over the side peaks at $\pm 7 \mathrm{fs}$ is about $10: 1$. The instantaneous bunching reaches nearly $70 \%$ with relatively little contrast. Snapshots of the Wigner transform of the on-axis far field (Fig. 5) again indicate highly localized emission in time and wavelength, together with a characteristic positive $d \lambda / d t$ chirp. Th central wavelength of $8.3 \mathrm{~nm}$ is sufficiently far from the input seed wavelength that the latter can be easily removed by wideband monochromatization to improve signal to background ratios. The magnitude of the chirp, $\sim 5.2 \mathrm{~nm} /$ fs agrees well quantitatively with the expected value

$$
\frac{d \lambda}{d t}=c \lambda_{w} \frac{d \ln a_{w}}{d z}\left(\frac{a_{w}^{2}}{1+a_{w}^{2}}\right) \approx 5.4 \mathrm{~nm} / \mathrm{fs}
$$

Figure 6 plots the instantaneous output radiation power and the time-resolved power loss by the ebeam. The latter curve indicates that the majority of the extracted power comes from the portion of the ebeam with the "correct" energy gradient as given by Eq. 2 while the portion toward the head separated by $\sim \lambda_{M} / 2$ that has a reverse sign in $\mathrm{d} \gamma / d t$ is actually significantly accelerated (as indicated by a negative energy loss). 


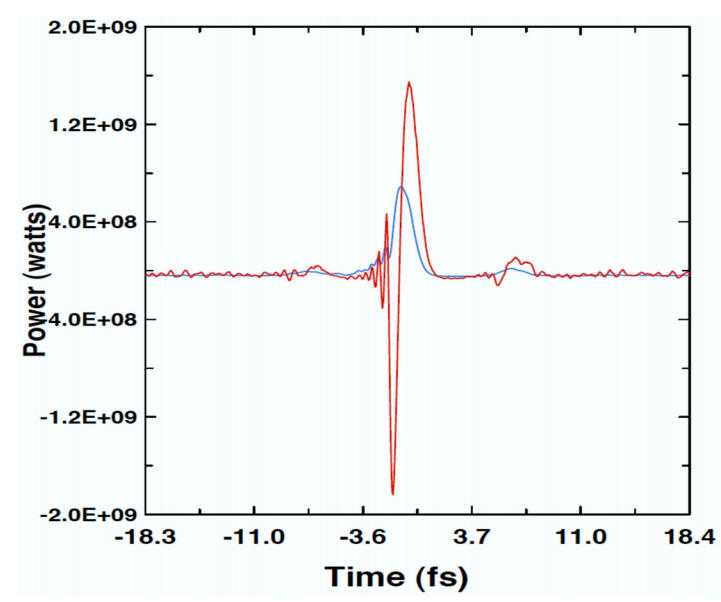

Fig. 6. Output $(z=8 \mathrm{~m})$ radiation power (blue curve) and net time-resolved power lost by the electron beam (red curve). Both curves are displaced -50 MW.

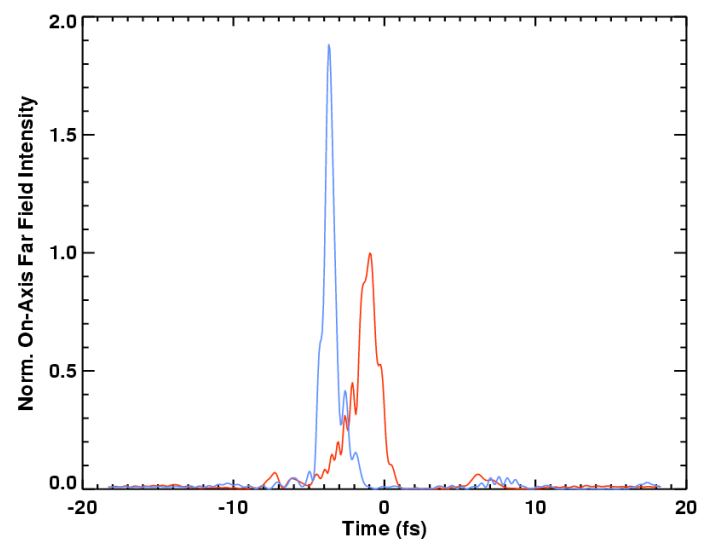

Fig. 7. Optimal compression (blue curve) of the chirped output (red curve) of the 8-nm seeded case shown in the previous three figures.

\section{Pulse Compression and Chirp Control}

The presence of a linear chirp in the radiation output implies that the pulse can be compressed via standard methods; Fig. 7 shows compression results for the 8-nm seeded case (e.g., Fig. 4) in which the peak power nearly doubles. Somewhat smaller compression ratios (e.g., a factor of 1.6) have also been obtained for SASE cases at $8 \mathrm{~nm}$.

Although Saldin et al. [7] chose a positive $d a_{w} / d z$ and negative $d \gamma / d t$ to optimize the gain contrast to the non-energy-modulated portion of the electron pulse, by reversing the sign of each one can still produce a well-confined (in time and wavelength) radiation pulse. As an initial numerical test, we reversed the sign of both $\mathrm{d} \gamma / d t$ by changing $\phi_{M}$ to zero (see Eq. 3) and the undulator taper to $-3.2 \%$

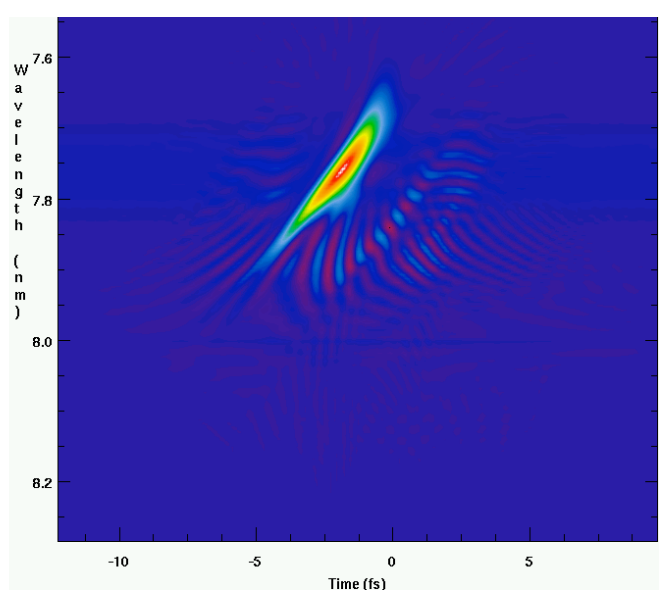

Fig. 8. Wigner transform of the far field, on-axis emission at $z=8 \mathrm{~m}$ for a seeded case with a "normal" (decreasing $\left.a_{w}\right)$ taper and positive $d \gamma / d t$ optical laser-induced energy modulation.

/ $10 \mathrm{~m}$. As displayed in Fig. 8, the changes lead to a blueward shift of $\langle\lambda\rangle$ with $z$ and a negative $d \lambda / d t$ for the chirp. The observed value, $5.2 \mathrm{~nm} / \mathrm{fs}$, agrees well with the predicted value of $5.1 \mathrm{~nm} / \mathrm{fs}$. As before, simple monochromatization would eliminate the temporally "long" seed signal at $8.0 \mathrm{~nm}$.

Consequently, between manipulation of the terms in Eqs. (2) and (5) and possible optical compression, users may have a number of intriguing "knobs" to vary the sign and magnitude of the output chirp.

\section{References}

[1] W. Ackermann et al., Nature Photonics, 1, 336 (2007).

[2] Linac Coherent Light Source (LCLS) Conceptual Design Report, SLAC Rpt. SLAC-R-593 (2002).

[3] P. Emma et al. , Phys. Rev. Lett., 92, 074801 (2004)

[4] A.A. Zholents and W.M. Fawley, Phys. Rev. Lett., 92, 224801 (2004).

[5] E.L. Saldin, E.A. Schneidmiller, and M.V. Yurkov, Opt. Commun., 237, 153 (2004).

[6] E.L. Saldin, E.A. Schneidmiller, and M.V. Yurkov, Opt. Commun., 239, 161 (2004).

[7] E.L. Saldin, E.A. Schneidmiller, and M.V. Yurkov, Phys. Rev. ST Accel. Beams, 9, 050702 (2006).

[8] A.A. Zholents and G. Penn, Phys. Rev. ST Accel. Beams, 8, $050704(2005)$

[9] H.-D. Nuhn, Nucl. Instr. Meth. Phys. Res. , A429, 249256 (1999); also SLAC-PUB-7912.

[10] Z. Huang and G. Stupakov, Phys. Rev. ST Accel. Beams, 8, $040702(2005)$.

[11] R. Bonifacio, C. Pellegrini, and L. Narducci, Opt. Commun., 50, 373 (1984).

[12] W. M. Fawley, LBNL Tech. Rpt. No. LBNL-49625Rev. 1 (2004); also SLAC Rpt. LCLS-TN-04-3

[13] M. Xie, Nucl. Instr. Meth. Phys. Res., A445 59 (2000). 